\title{
How to quantify coughing: correlations with quality of life in chronic cough
}

\author{
A. Kelsall*, , S. Decalmer*, D. Webster*, N. Brown*, K. McGuinness*,", \\ A. Woodcock**\# and J. Smith*
}

ABSTRACT: Different methods are used for quantifying coughing in sound recordings, but as yet no method has been shown to be more valid than any other. In the present study, the relationships between three different units of cough were examined and their ability to predict subjective ratings of cough and cough-related quality of life were evaluated.

In total, 70 subjects (mean \pm SD age $55 \pm 11.7$ yrs, 51 (73\%) females) with chronic unexplained cough (median duration 4.8 yrs, interquartile range 2.5-10.1 yrs) performed fully ambulatory 24-h sound recordings, which were manually counted by trained observers and quantified by 1) explosive phases, 2) cough seconds and 3) cough epochs. Subjects also completed cough visual analogue scales (VAS) and the Leicester Cough Questionnaire (LCQ).

All units of cough were strongly correlated; explosive phases and cough seconds correlated slightly more strongly than cough seconds with cough epochs or explosive phases with cough epochs. LCQ scores correlated moderately with explosive phases and seconds; epochs correlated slightly less well. Cough VAS scores showed a similar pattern.

Explosive phases and seconds are interchangeable units of cough, moderately related to subjective measures and cough-related quality of life; epochs are a less satisfactory alternative.

KEYWORDS: Ambulatory cough monitoring, chronic cough, cough epochs, cough seconds, explosive phases

$\mathrm{T}$ he assessment of cough, both in clinical practice and in clinical research, has been impeded by a lack of valid tools. In recent years, cough assessment has been improved by the development of objective cough monitoring systems [1-4] and cough-specific quality-of-life questionnaires $[5,6]$. Ambulatory sound recording systems are increasingly used to measure coughing objectively, but there is no agreement about the best method for quantifying the recorded events. Indeed, many studies to date have failed to describe how cough is defined or quantified [7]. The European guidelines for the assessment of cough state: "there is little to commend any particular method of quantifying cough over any other" [8].

Acoustic cough waveforms are generally described as comprising three phases [9]: an "explosive" phase, produced by sudden opening of the glottis; an "intermediate" phase as the cough sound decays; and sometimes an additional "glottal" or "voiced" phase, produced by a partial closure of the vocal cords (fig. 1). In contrast, sound recordings of spontaneous coughing find a much wider variety of patterns. In disease, prolonged series of explosive phases tend to occur (fig. 2), either after a single breath or with several breaths interspersed; these are often referred to as cough "epochs", "peals", "bouts" or "attacks" [7]. Consequently, coughing is commonly quantified by counting either explosive phases or epochs. A novel method was recently described for quantifying coughing: "cough seconds" [10-13]. The number of seconds containing at least one explosive phase is counted, giving an estimate of the amount of time spent coughing.

Depending on the unit of cough, very different results can be obtained from the same sound recording. In figure 2, a series of cough waveforms can be quantified either as four explosive phases, two cough seconds or a single cough epoch. In small groups of patients with cystic fibrosis, chronic obstructive pulmonary disease, asthma, chronic cough or interstitial lung disease, there is a linear relationship between cough
AFFILIATIONS

*Respiratory Research Group, University of Manchester, and ${ }^{\#}$ North West Lung Centre, University Hospital of South Manchester NHS Foundation Trust, Manchester, UK.

CORRESPONDENCE

A. Kelsall

North West Lung Research Centre Wythenshawe Hospital

Southmoor Road Wythenshawe Manchester M23 9LT UK

Fax: 441612915057

E-mail: Angela.Kelsall@

manchester.ac.uk

Received:

August 062007

Accepted after revision:

February 042008

\section{SUPPORT STATEMENT}

This study received financial support from the Moulton Charitable Trust (Sevenoaks, UK).

STATEMENT OF INTEREST

None declared. 
seconds and explosive phases [14]. The relationships between all the different units of cough in use have not been defined but their understanding would facilitate comparison of studies where the units differ.

The aim of the present study was to compare different methods for quantifying coughing in subjects with chronic
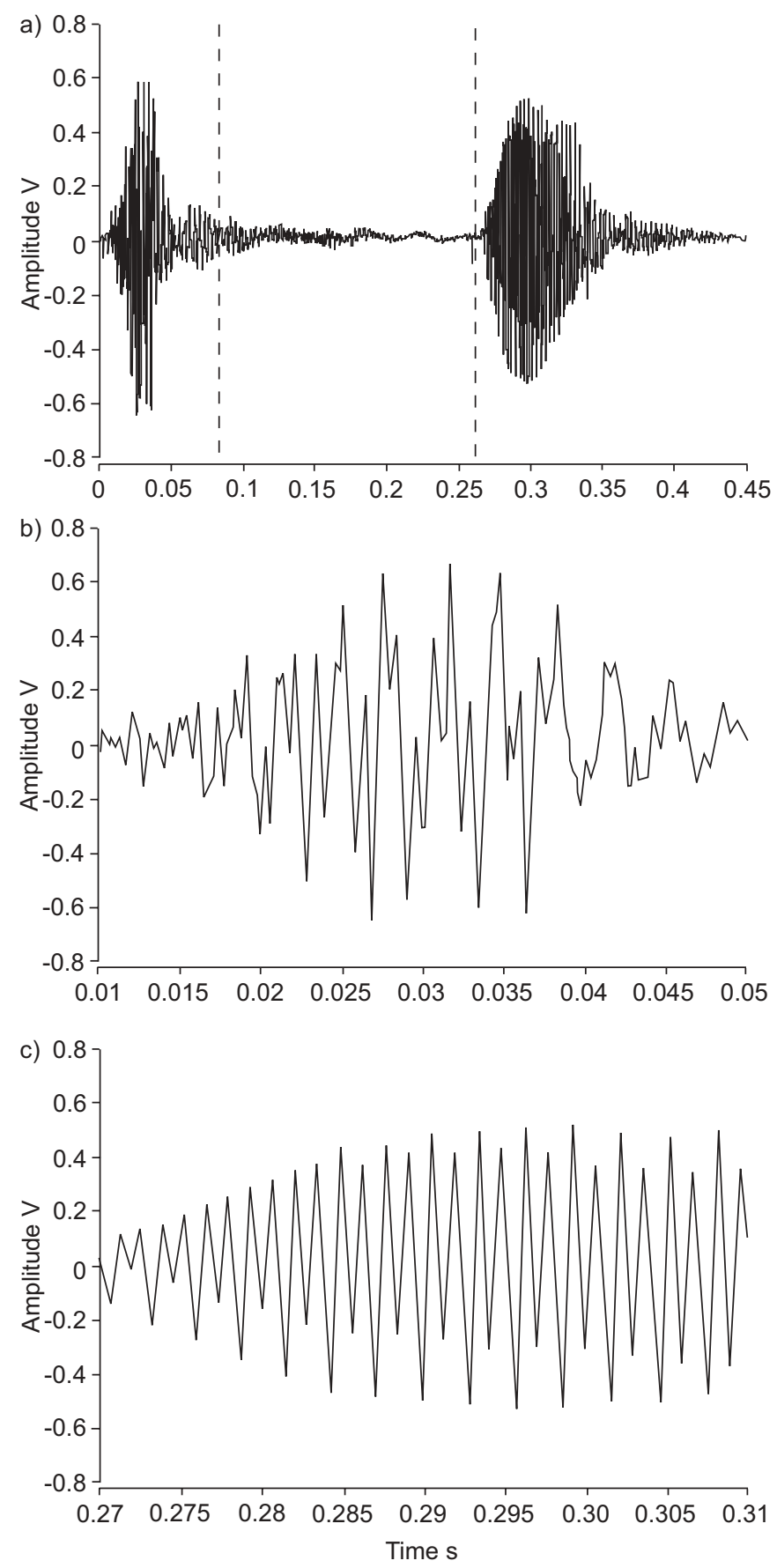

FIGURE 1. a) Typical cough sound waveform divided into the three acoustic phases called the explosive (left), intermediate (centre) and voiced (right) phases. b) The explosive phase on an expanded timescale, demonstrating the irregular, noise-like appearance. c) The voiced phase on an expanded timescale, showing its contrasting regular, periodic appearance. cough. Additionally, the validity of these different cough units was tested by examining correlations with the patients' perception of cough, measured by the cough visual analogue scale (VAS) and cough-specific quality of life.

\section{METHODS AND MATERIALS}

\section{Subjects}

Patients with chronic unexplained cough ( $>8$ weeks' duration) were recruited from a tertiary referral clinic (North West Lung Centre, Wythenshawe Hospital, Manchester, UK). Current smokers and those who had stopped smoking for $<6$ months were excluded from the study. Approval was granted by the Local Research Ethical Approval board (South Manchester, UK) and written consent obtained.

\section{Cough recordings}

Fully ambulatory 24-h sound recordings were made, using a lapel microphone with either a modified MP3 player (IAudio; Cowon Systems Inc., Seoul, South Korea; $\mathrm{n}=31$ ) or a validated custom-built recording device (Vitalograph Ltd, Buckingham, UK; $n=39$ ). Participants were encouraged to continue their normal daily routine over the monitoring period. Recordings were downloaded to a PC and cough waveforms were quantified by a trained observer using an audiovisual display (CoolEdit2000 (IM); Syntrillium Software Corporation, Phoenix, AZ, USA). Excellent inter- and intra-subject agreement has been found for quantification of cough seconds, explosive phases and cough epochs [15-18]. For each recording, coughs were quantified according to the following three methods (fig. 2 and online supplementary data).

\section{Explosive phases}

The explosive phase is the characteristic sound recognised as a cough. The irregular, noise-like waveform was readily differentiated from voiced phases, which were audibly voice-like and had a regular (periodic) waveform (fig. 1).

\section{Cough seconds}

The number of seconds containing at least one explosive phase were counted.

\section{Cough epochs}

The number of periods of continuous coughing, with pauses of $<2$ s, were counted. The audiovisual display of the sound recordings showed the waveforms plotted against time, allowing measurement of pauses. Cough epochs defined as breaths containing at least one explosive phase could not be examined using the present system, as it was not validated for detecting breath sounds.

\section{Subjective measures of cough}

After each 24-h recording, subjects completed a cough VAS for both day and night, i.e. a 100-mm linear scale, where the extremes were marked "no cough" and "worst cough ever". Cough-related quality of life was measured using the Leicester Cough Questionnaire (LCQ), a validated questionnaire comprising 19 questions [5]. Scores ranged 3-21, with higher scores representing better quality of life. 

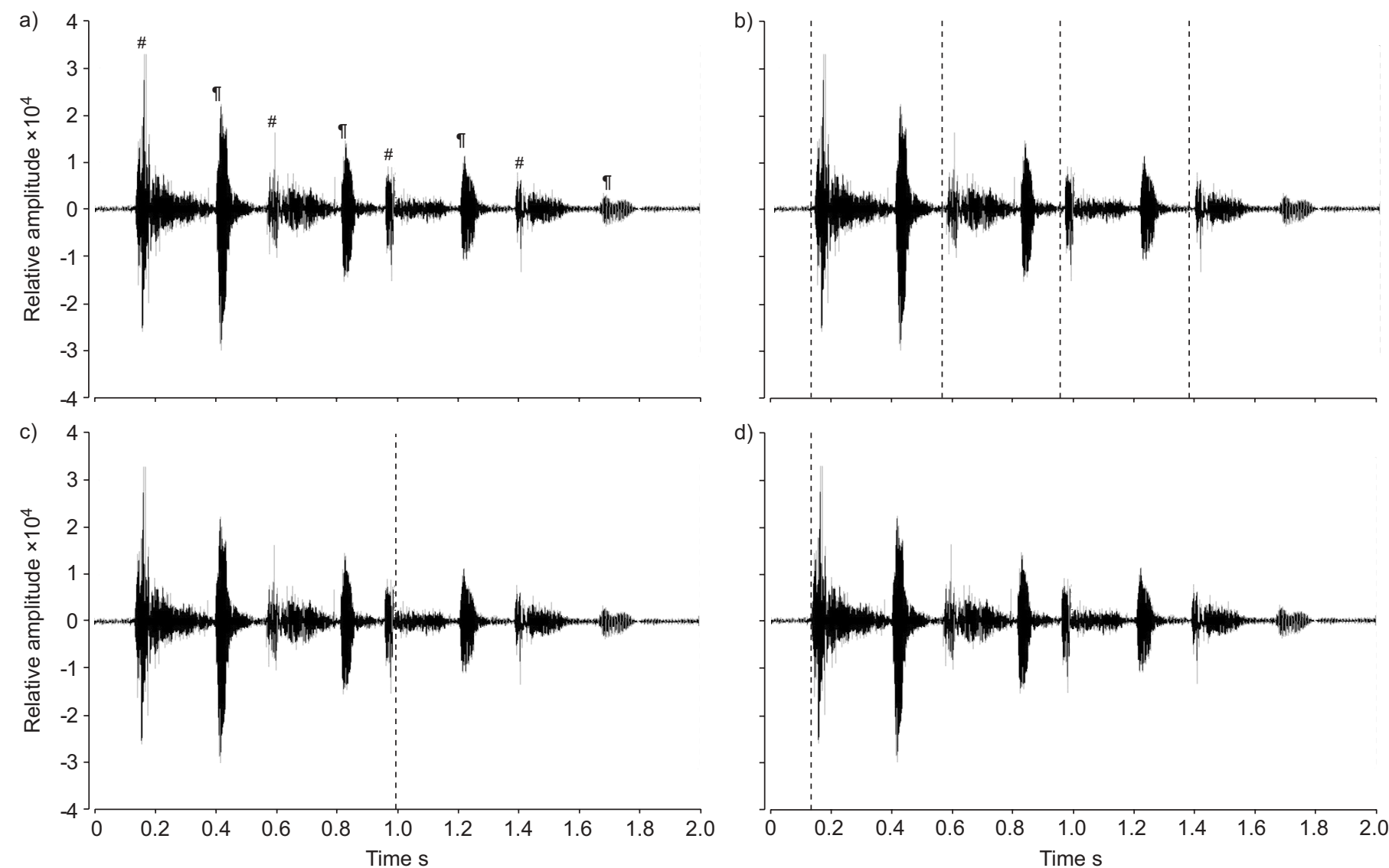

FIGURE 2. Comparison of different methods for quantifying cough sound recordings. a) Explosive (\#) and voiced (") phases in cough waveforms. b) Counting four explosive phases. c) Counting two cough seconds. d) Counting one cough epoch (for further details see the online supplementary data).

\section{Statistical analysis}

Day, night and overall cough rates, for all three methods, were positively skewed; logarithmic transformation (base 10) normalised the distributions. As night-time VAS and night-time cough rates contained zero values, one unit was added to each data point to allow logarithmic transformation.

\section{RESULTS}

\section{Subjects}

In total, 70 subjects (mean \pm SD age $55 \pm 11.7$ yrs, 51 (73\%) females) with chronic unexplained cough (median duration $4.8 \mathrm{yrs}$, interquartile range (IQR) 2.5-10.1 yrs) were studied. Of these subjects, two did not complete the cough VAS. TABLE 1 Diurnal variation in cough rate for the three units
of cough

\begin{tabular}{lccc} 
& 24-h & Day & Night \\
\hline Explosive phases $\cdot \mathbf{h}^{-1}$ & $15.9(8.6-23.0)$ & $19.3(11.4-33.6)$ & $4.5(1.3-10.6)$ \\
${\text { Cough seconds } \cdot \mathbf{h}^{-1}}^{\text {Cough epochs } \cdot \mathbf{h}^{-\mathbf{1}}}$ & $12.2(6.8-18.1)$ & $16.3(9.2-26.2)$ & $3.4(1.0-9.1)$ \\
& $5.1(2.9-7.1)$ & $6.4(3.6-9.5)$ & $1.4(0.4-3.2)$ \\
\hline \\
Data are presented as median (interquartile range).
\end{tabular}

\section{Cough quantification}

The median cough rates for the three different cough units are given in table 1. All three methods for quantifying cough were highly correlated (table 2). The strongest correlation was between explosive phases and cough seconds (shared variance $r^{2}=0.98$ ), while the correlations for each of these measures with cough epochs were less strong (cough seconds and cough epochs $\mathrm{r}^{2}=0.84$; explosive phases and cough epochs $\mathrm{r}^{2}=0.80$; table 2 and fig. 3).

The median (IQR) epoch length was 2.9 (2.1-3.6) explosive phases epoch ${ }^{-1}$. There was no difference between the median epoch length for day or night (2.7 (2.0-3.7) versus 3.1 (2.1-4.2), respectively; $\mathrm{p}=0.12$ ).

\begin{tabular}{|c|c|c|c|c|c|}
\hline \multirow[t]{3}{*}{ TABLE 2} & \multicolumn{5}{|c|}{$\begin{array}{l}\text { Pearson correlations between different units of } \\
\text { cough }\end{array}$} \\
\hline & & \multicolumn{2}{|c|}{ log cough seconds } & \multicolumn{2}{|c|}{ log cough epochs } \\
\hline & & r coefficient & p-value & r coefficient & p-value \\
\hline \multirow{2}{*}{\multicolumn{2}{|c|}{$\begin{array}{l}\text { log explosive phases } \\
\text { log cough seconds }\end{array}$}} & 0.99 & $\leqslant 0.001$ & 0.90 & $\leqslant 0.001$ \\
\hline & & $\mathrm{N} / \mathrm{A}$ & & 0.92 & $\leqslant 0.001$ \\
\hline
\end{tabular}



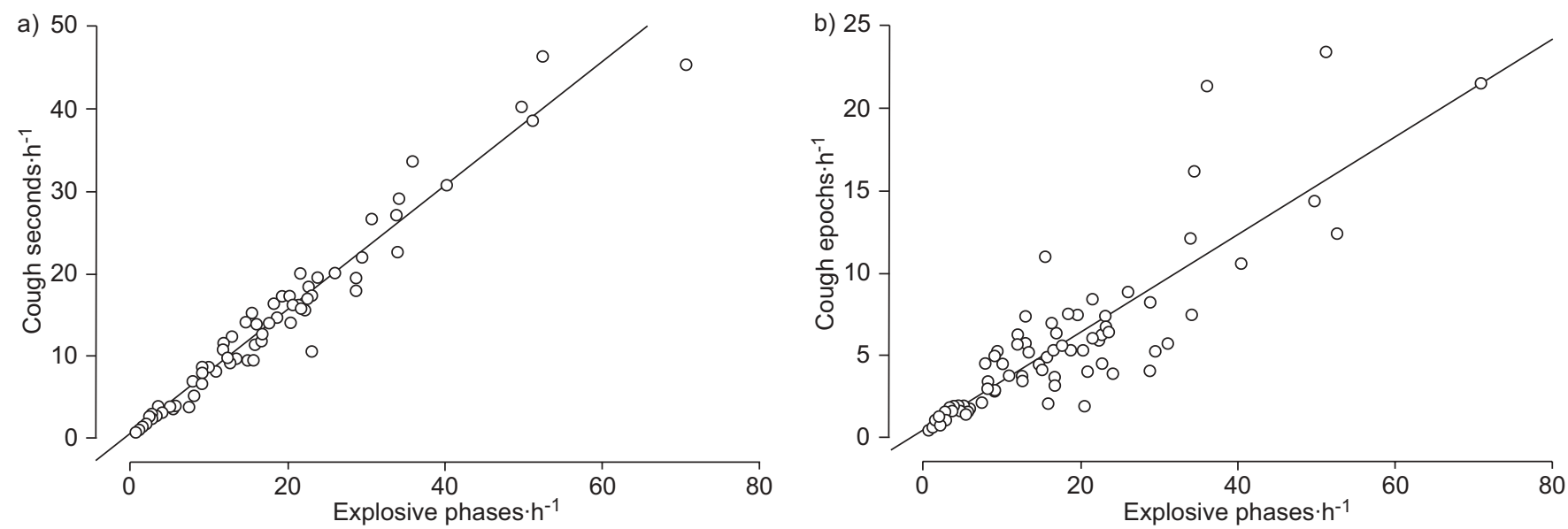

FIGURE 3. Correlations between a) cough seconds and explosive phases and b) cough epochs and explosive phases.

\section{Subjective measures and quality of life}

The mean \pm SD total LCQ score was $12.3 \pm 3.8$, the median (IQR) daytime VAS score was $40.0(21.5-62.8) \mathrm{mm}$ and the night-time VAS score was $18.0(5.3-45.8) \mathrm{mm}$. Over $24 \mathrm{~h}$, the rates of explosive phases and cough seconds had identical correlation coefficients with the LCQ, explaining $28 \%$ of the variance (table 3). Cough epochs correlated slightly less strongly and explained $21 \%$ of the variance. Correlations between the VAS and cough units showed a similar pattern.

\section{DISCUSSION}

The present study is the first to compare the three commonest methods for quantifying coughs during ambulatory monitoring. The measurements of explosive phases and cough seconds were extremely strongly correlated with one another, sharing $98 \%$ of their variance and, thus, being virtually interchangeable. Moreover, both measures were similarly related to coughrelated quality of life and the patients' subjective estimate of cough severity. Cough epochs were less closely related to either explosive phases or cough seconds, sharing $80 \%$ and $84 \%$ of variance, respectively, and were less well correlated with patients' reports. Quantification in terms of epochs effectively ignored epoch length; the poorer correlations with cough quality of life and VAS imply that epoch length was important to patients.

Cough-related quality of life and VAS are becoming widely used to assess patients with cough. While all units of cough were significantly correlated with both subjective measures, the correlation coefficients were at best moderate, so the majority of the variance in these scores is not explained by objective cough frequency. It is inevitable that subjective scores and quality of life are influenced by much more than just the number of coughs, regardless of the units used. The intensity or effort involved in coughing is ignored, but it is likely that the most intense coughs have the greatest impact for patients. Anxiety and depression are

\section{TABLE 3 Correlations between different units of cough and subjective measures}

\begin{tabular}{|c|c|c|c|c|c|c|}
\hline & \multicolumn{2}{|c|}{ LCQ } & \multicolumn{2}{|c|}{ Day VAS } & \multicolumn{2}{|c|}{ Night VAS } \\
\hline & $r$ coefficient & p-value & $r$ coefficient & p-value & $r$ coefficient & p-value \\
\hline \multicolumn{7}{|c|}{ log explosive phases } \\
\hline 24-h & -0.53 & $\leqslant 0.001$ & & & & \\
\hline Day & -0.49 & $\leqslant 0.001$ & 0.45 & $\leqslant 0.001$ & & \\
\hline 24-h & -0.53 & $\leqslant 0.001$ & & & & \\
\hline Day & -0.50 & $\leqslant 0.001$ & 0.44 & $\leqslant 0.001$ & & \\
\hline Night & -0.35 & 0.003 & & & 0.64 & $\leqslant 0.001$ \\
\hline \multicolumn{7}{|c|}{ log cough epochs } \\
\hline 24-h & -0.46 & $\leqslant 0.001$ & & & & \\
\hline Day & -0.36 & 0.002 & 0.40 & 0.001 & & \\
\hline
\end{tabular}

LCQ: Leicester Cough Questionnaire; VAS: visual analogue scale. Note that negative correlations are present for the LCQ as lower scores and worse quality of life were associated with higher cough rates. 
common in chronic cough $[19,20]$ and may also be important. It has recently been shown that anxiety scores significantly predict cough-related quality of life [21]. Vigilance and recall may also differ between patients. Cough is an episodic symptom, and some subjects may be better than others at recalling the frequency of cough and associated disruption; this is likely to be related to how much attention they pay to the symptom.

These issues highlight the limitations of using subjective scores or objective quantification of coughing alone. A comprehensive assessment of cough requires both measures. In future, methods that would allow an objective measure of cough intensity would be a valuable addition to cough monitoring.

One limitation of the present study is that only cough epochs defined as continuous coughing, with pauses of $<2$ s, could be examined. When monitoring coughing from sound alone, it was not possible to count cough epochs defined as a single expiratory effort with multiple explosive phases. A simultaneous measure of respiratory rate would be needed, which may give different results.

In conclusion, the current study showed that different units of cough quantification were remarkably highly correlated. Explosive phases and cough seconds were correlated sufficiently closely to be interchangeable, and correlated moderately with cough-related quality of life and subjective assessment of cough severity. Cough epochs were a less satisfactory alternative.

\section{REFERENCES}

1 Coyle MA, Keenan DB, Henderson LS, et al. Evaluation of an ambulatory system for the quantification of cough frequency in patients with chronic obstructive pulmonary disease. Cough 2005; 1: 3.

2 Matos S, Birring SS, Pavord ID, Evans DH. Detection of cough signals in continuous audio recordings using hidden Markov models. IEEE Trans Biomed Eng 2006; 53: 1078-1083.

3 McGuinness K, Kelsall A, Lowe J, Woodcock A, Smith JA. Automated cough detection: a novel approach. Am J Respir Crit Care Med 2007; 175: A381.

4 Barry SJ, Dane AD, Morice AH, Walmsley AD. The automatic recognition and counting of cough. Cough 2006; 2: 8.

5 Birring SS, Prudon B, Carr AJ, Singh SJ, Morgan MD, Pavord ID. Development of a symptom specific health status measure for patients with chronic cough: Leicester Cough Questionnaire (LCQ). Thorax 2003; 58: 339-343.
6 French CT, Irwin RS, Fletcher KE, Adams TM. Evaluation of a cough-specific quality-of-life questionnaire. Chest 2002; 121: 1123-1131.

7 Fontana GA, Widdicombe J. What is cough and what should be measured? Pulm Pharmacol Ther 2007; 20: 307-312.

8 Morice AH, Fontana GA, Belvisi MG, et al. ERS guidelines on the assessment of cough. Eur Respir J 2007; 29: 1256-1276.

9 Korpás J, Sadlonová J, Vrabec M. Analysis of the cough sound: an overview. Pulm Pharmacol 1996; 9: 261-268.

10 Decalmer S, Webster D, Kelsall A, McGuinness K, Woodcock A, Smith J. Chronic cough: how do cough reflex sensitivity and subjective assessments correlate with objective cough counts during ambulatory monitoring? Thorax 2007; 62: 329-334.

11 Smith J, Owen E, Earis J, Woodcock A. Effect of codeine on objective measurement of cough in chronic obstructive pulmonary disease. J Allergy Clin Immunol 2006; 117: 831-835.

12 Smith J, Owen E, Earis J, Woodcock A. Cough in COPD: correlation of objective monitoring with cough challenge and subjective assessments. Chest 2006; 130: 379-385.

13 Smith JA, Owen EC, Jones AM, Dodd ME, Webb AK, Woodcock A. Objective measurement of cough during pulmonary exacerbations in adults with cystic fibrosis. Thorax 2006; 61: 425-429.

14 Smith J. Ambulatory methods for recording cough. Pulm Pharmacol Ther 2007; 20: 313-318.

15 Smith J. The objective measurement of cough. PhD thesis. University of Manchester, Manchester, UK, 2004.

16 Hsu JY, Stone RA, Logan-Sinclair RB, Worsdell M, Busst CM, Chung KF. Coughing frequency in patients with persistent cough: assessment using a 24-hour ambulatory recorder. Eur Respir J 1994; 7: 1246-1253.

17 Munyard P, Busst C, Logan-Sinclair R, Bush A. A new device for ambulatory cough recording. Pediatr Pulmonol 1994; 18: 178-186.

18 Hamutcu R, Francis J, Karakoc F, Bush A. Objective monitoring of cough in children with cystic fibrosis. Pediatr Pulmonol 2002; 34: 331-335.

19 Dicpinigaitis PV, Tso R, Banauch G. Prevalence of depressive symptoms among patients with chronic cough. Chest 2006; 130: 1839-1843.

20 McGarvey LP, Carton C, Gamble LA, et al. Prevalence of psychomorbidity among patients with chronic cough. Cough 2006; 2: 4.

21 Decalmer S, Kelsall A, McGuinness K, Woodcock A, Smith JA. Anxiety, depression and quality of life measures in patients with chronic persistent cough. Thorax 2007; 62: Suppl. 3, A108. 